\title{
Control glucémico en pacientes con diabetes e infección crónica por VHC, tratados con antivirales de acción directa
}

\section{Glycemic control in patients with diabetes and chronic HCV infection, treated with direct-acting antivirals}

\author{
Juan C. Aguilar-de La O ${ }^{1 *}$, Aleida Bautista-Santos ${ }^{2}$ y Juan C. Anda-Garay ${ }^{1}$ \\ 'Departamento de Medicina Interna; ${ }^{2}$ Departamento de Gastroenterología. Unidad Médica de Alta Especialidad, Centro Médico \\ Nacional Siglo XXI Dr. Bernardo Sepúlveda Gutiérrez, Instituto Mexicano del Seguro Social, Ciudad de México, México
}

\section{RESUMEN}

\begin{abstract}
Antecedentes: La infección por virus de hepatitis C (VHC) se ha asociado a diabetes mellitus y/o resistencia a la insulina. El tratamiento con antivirales de acción directa (AAD) logra la respuesta viral sostenida (RVS) en $>95 \%$ y se ha observado mejoría en el control glucémico posterior al tratamiento. Material y métodos: Se incluyeron personas mayores de 18 años, con diagnóstico de infección crónica por VHC y diabetes mellitus tipo 2 que hayan recibido tratamiento con AAD con RVS y que cuenten con glucosa sérica y hemoglobina glucosilada (HbA1c) basal y postratamiento. Resultados: Se incluyeron un total de 13 pacientes $(30.8 \%$ hombres y $69.2 \%$ mujeres). La RVS fue del $100 \%$. Respecto al control glucémico, se observó mejoría en los niveles de glucosa sérica posterior al tratamiento (glucosa previa $166.15 \pm 66.67 \mathrm{mg} / \mathrm{dl}$ vs. postratamiento $123.31 \pm 26.97 \mathrm{mg} / \mathrm{dl})(\mathrm{p}<0.046)$ y niveles de HbA1c (previa $7.62 \pm 2.32 \%$ vs. postratamiento $6.86 \pm 1.56 \%$ ) $(p=0.062)$. Conclusión: Nuestro estudio en población mexicana es concordante con lo reportado en estudios internacionales, debido a que el $100 \%$ de los participantes tratados con AAD presentó RVS, generando un impacto positivo en el control glucémico.
\end{abstract}

Palabras clave: Infección crónica por VHC. Diabetes mellitus tipo 2. Control glucémico. Antivirales de acción directa.

\begin{abstract}
Background: Infection with the hepatitis C virus (HCV) has been associated with diabetes mellitus and / or insulin resistance. Treatment with direct-acting antivirals (DAAs) achieves a sustained viral response (SVR) in $>95 \%$, and an improvement in glycemic control has been observed after treatment. Material and methods: People over 18 years of age with a diagnosis of chronic HCV infection and type 2 diabetes mellitus who had received DAA treatment with SVR and had basal and post-treatment serum glucose and glycated hemoglobin were included. Results: 13 patients were included (30.8\% men and 69.2\% women). The sustained viral response (SVR) was $100 \%$. Regarding glycemic control, an improvement was observed in serum glucose levels after treatment (prior glucose $166.15 \mathrm{mg} / \mathrm{dl} \pm 66.67$ vs. post-treatment $123.31 \mathrm{mg} / \mathrm{dl} \pm 26.97)(\mathrm{p}=<0.046)$ and glycosylated hemoglobin levels ( $\mathrm{HbA} 1 \mathrm{c}$ above $7.62 \% \pm 2.32$ vs. post-treatment $6.86 \% \pm 1.56)(p=0.062)$. Conclusion: Our study in the Mexican population is in agreement with that reported in international studies, since $100 \%$ of the participants treated with DAAs presented SVR, generating a positive impact on glycemic control.
\end{abstract}

Keywords: Chronic HCV infection. Type 2 diabetes mellitus. Glycemic control. Direct-acting antivirals.
Correspondencia:

*Juan C. Aguilar-de La O

E-mail: drjuancarlosao@gmail.com
Fecha de recepción: 23-02-2021

Fecha de aceptación: 14-07-2021

DOI: 10.24875/RME.21000015
Disponible en internet: 22-02-2022

Rev Mex Endocrinol Metab Nutr. 2022;9:20-6

2462-4144 / @ 2021 Sociedad Mexicana de Nutrición y Endocrinología, AC. Publicado por Permanyer. Este es un artículo open access bajo la licencia CC BY-NC-ND (http://creativecommons.org/licenses/by-nc-nd/4.0/). 


\section{INTRODUCCIÓN}

La infección por virus de hepatitis C (VHC) se ha convertido en un problema de salud importante. La Organización Mundial de la Salud estima que aproximadamente el 3\% de la población mundial (150-170 millones de personas) viven con infección crónica, dentro de las complicaciones que más destacan son la cirrosis hepática y el carcinoma hepatocelular. Sin embargo, dicha entidad se ha asociado a enfermedades cardiovasculares, renales, del sistema nervioso central y metabólicas, de las cuales destacan un mayor riesgo de diabetes mellitus o resistencia en la insulina?.

En México la diabetes mellitus y la enfermedad hepática crónica son muy prevalentes, en el 2008 representaron la primera y cuarta causa de mortalidad general, respectivamente ${ }^{2}$. La prevalencia de la infección por VHC en México es del $0.4 \%$ y los datos acerca de la asociación de ambas entidades en nuestro país son escasas ${ }^{2}$. Hasta el momento se reconocen seis genotipos del VHC, del 1 al 6 . En nuestro país predomina el genotipo 1 y de los subtipos, el $1 \mathrm{~b}$ es el de mayor prevalencia ${ }^{3}$.

\section{VIRUS DE HEPATITIS C Y ALTERACIONES EN LA GLUCOSA}

En el caso de diabetes mellitus tipo 2 (DM2), la resistencia a la insulina es parte del sustrato fisiopatológico de la enfermedad, por lo que si se agrega la resistencia a la insulina mediada por el VHC se incrementa el riesgo de desarrollar DM2 en esta población ${ }^{4}$. La prediabetes es cuatro veces más frecuente en personas con VHC, siendo factores predictores para su desarrollo la edad avanzada y niveles elevados de alanina aminotransferasa ${ }^{5}$. Se han reportado factores de riesgo para el desarrollo de diabetes en pacientes con VHC, dentro de los cuales se encuentran el sexo masculino, edad mayor a 40, índice de masa corporal (IMC) elevado, categoría F4 en elastografía hepática, el tiempo de duración de la infección, la respuesta a la terapia previa y antecedentes familiares de diabetes ${ }^{6}$.
EFECTO DE LOS ANTIVIRALES DE ACCIÓN DIRECTA SOBRE EL CONTROL GLUCÉMICO

El tratamiento antiviral de VHC puede conducir a la mejora del metabolismo de la glucosa, principalmente en pacientes que lograron respuesta viral sostenida (RVS) ${ }^{7}$. Los pacientes con infección crónica por VHC tienen 3.8 veces más probabilidades de padecer diabetes que los sujetos con VHC negativo, aun no está bien definido si el causante de la resistencia a la insulina es el VHC o la respuesta inflamatoria del huésped, lo cual contribuye al riesgo de diabetes a largo plazo ${ }^{8}$. Algunos ensayos clínicos han mostrado mejoría en el control glucémico en personas con diabetes y sin diabetes posterior al tratamiento con antivirales de acción directa $(A A D)^{9}$.

En un estudio realizado en el 2017 por Hum, et al., publicado por la Asociación Americana de Diabetes (ADA), se incluyeron 2,435 pacientes con diabetes que se sometieron a tratamiento antiviral directo sin interferón ni ribavirina para la infección crónica de VHC; se comparó a los pacientes que alcanzaron RVS con los que no lo hicieron y se encontró que el grupo que alcanzó RVS fueron menos propensos a recibir medicamentos antidiabéticos (74.8 vs. $78 \%$ ) o insulina (41.3 vs. $49.8 \%$ ), menor probabilidad de tener cirrosis (35.3 vs. $54.5 \%$ ) y cirrosis descompensada ( 9.3 vs. $20 \%$ ). La caída en el nivel promedio de hemoglobina glucosilada ( $\mathrm{HbA1c}$ ) después del tratamiento fue mayor en el primer grupo (del 7.2 al 6.82\%), que en aquellos que el tratamiento no fue exitoso (del 7.27 al $7.08 \%$, con una caída promedio del $0.19 \pm 1.3 \%$ ) produciendo una diferencia media del $-0.18 \%$. Concluyeron que la erradicación del VHC con AAD conduce a un mejor control glucémico en personas con diabetes ${ }^{10}$. Los efectos secundarios de la terapia con AAD son mínimos y la mayoría son bien tolerados, los más frecuentes son: cefalea, náuseas y mareo dependiendo del esquema indicado; uno de ellos puede ser la hipoglucemia ${ }^{11}$.

En los últimos años se desarrollaron los AAD con los cuales se han logrado altas tasas de curación, lo cual se define con el logro de RVS al término del 
tratamiento. Esto ha llevado a mejoría del control glucémico en personas con diabetes y sin diabetes, pero con mayor significado clínico en el primer grupo ${ }^{12}$.

Por lo tanto, al carecer de estudios en nuestro país que identifiquen la asociación entre diabetes y VHC, los objetivos de nuestro estudio fueron demostrar la mejoría en el control glucémico después del tratamiento antiviral directo, así como las características clínicas y bioquímicas de los pacientes con diabetes y VHC.

\section{MATERIALES Y MÉTODOS}

Se incluyeron pacientes que acudieron a la consulta externa del Hospital de Especialidades del Centro Médico Nacional Siglo XXI, de la Ciudad de México, del 1 de enero de 2019 al 1 de enero de 2020, incluyendo personas mayores de 18 años con infección crónica por VHC y diabetes que hayan recibido tratamiento con AAD (sofosbuvir/velpatasvir, sofosbuvir/velpatasvir más ribavirina y sofosbuvir/glecaprevir/pibrentasvir) que tuvieron RVS (carga viral de VHC negativa al término del tratamiento), que contaban en su expediente con glucosa sérica, $\mathrm{HbA} 1 \mathrm{c}$ y carga viral de VHC antes y después del tratamiento con AAD (12 semanas). Se excluyeron pacientes con anemia, enfermedad renal crónica con tasa de filtrado glomerular $<30 \mathrm{ml} / \mathrm{min} / 1.73 \mathrm{~m}^{2}$, quienes hayan recibido tratamiento antiviral en los últimos 12 meses. La estadística descriptiva se realizó con medidas de dispersión para las variables continuas y con proporciones para las variables categóricas. Las variables dicotómicas se analizaron con la prueba Chi cuadrada. Para mostrar diferencias se utilizó la t de Student. El análisis estadístico se realizo con el paquete SPSS versión 25 .

\section{RESULTADOS}

Para evaluar el papel de las diferentes variables se realizó prueba de Kolmogorov-Smirnov para identificar la distribución de los datos, identificándose entre las variables una distribución normal, por lo
Tabla 1. Características basales de los pacientes con infección crónica por virus de hepatitis C (VHC) tratados con antivirales de acción directa (AAD) $(\mathrm{n}=13)$

\begin{tabular}{|c|c|}
\hline \multirow[t]{2}{*}{ Sexo; n (\%) } & Mujer: 9 (69.2) \\
\hline & Hombre: 4 (30.8) \\
\hline Edad (años); M & $60.54 \pm 16.59$ \\
\hline Peso $(\mathrm{kg}) ; \mathrm{M}$ & $69.9 \pm 16.35$ \\
\hline $\mathrm{IMC}\left(\mathrm{kg} / \mathrm{m}^{2}\right) ; \mathrm{M}$ & $27.57 \pm 5.69$ \\
\hline \multirow{2}{*}{$\begin{array}{l}\text { Adherencia al tratamiento } \\
\text { antidiabético; n (\%) }\end{array}$} & Sí: 11 (84.6) \\
\hline & No: 2 (15.4) \\
\hline \multirow{2}{*}{$\begin{array}{l}\text { Tratamiento antidiabético previo } \\
\text { AAD; } n(\%)\end{array}$} & Sí: 11 (84.6) \\
\hline & No: 2 (15.4) \\
\hline \multirow{2}{*}{$\begin{array}{l}\text { Tratamiento con insulina previo } \\
\text { AAD; } n(\%)\end{array}$} & Sí: $3(23.1)$ \\
\hline & No: 10 (76.9) \\
\hline Glucosa previo AAD; $(\mathrm{mg} / \mathrm{dl}) ; \mathrm{M}$ & $166.15 \pm 66.67$ \\
\hline HbA1c previo AAD (\%); M & $7.62 \pm 2.32$ \\
\hline $\begin{array}{l}\text { Infección crónica por VHC } \\
\text { (meses); M }\end{array}$ & $94.54 \pm 101.113$ \\
\hline $\begin{array}{l}\text { Carga viral de VHC al } \\
\text { diagnóstico }(\mathrm{UI} / \mathrm{ml}) ; \mathrm{M}\end{array}$ & $8,316,306 \pm 17,258,796.35$ \\
\hline \multirow[t]{5}{*}{ Genotipo; n (\%) } & 1 (sin subtipo): 1 (7.7) \\
\hline & 1a: $1(7.7)$ \\
\hline & $1 \mathrm{~b}: 1(7.7)$ \\
\hline & $2: 8(61.5)$ \\
\hline & $3: 1(7.7)$ \\
\hline \multirow[t]{3}{*}{ Child Pugh; n (\%) } & A: $9(69.2)$ \\
\hline & B: $2(15.4)$ \\
\hline & $C: 2(15.4)$ \\
\hline
\end{tabular}

HbA1c: hemoglobina glucosilada; IMC: índice de masa corporal; M: media.

que el análisis de las variables cuantitativas se realizó mediante prueba t de Student.

\section{Características demográficas de la población de estudio}

Se incluyeron un total de 13 pacientes con infección crónica por VHC y DM2, de los cuales cuatro (30.8\%) fueron hombres y nueve $(69.2 \%)$ mujeres; con un rango de edad de 27 a 81 años (media general de 60.54 años). Del resto de las características se encontraban con un rango de peso de 49.7 a $98.6 \mathrm{~kg}$ (media de $69.91 \mathrm{~kg}$ ); así también un rango de IMC de 22.2 a $39.78 \mathrm{~kg} / \mathrm{m}^{2}$ (media de $\left.28.16 \mathrm{~kg} / \mathrm{m}^{2}\right)$, de los cuales cinco (38.46\%) se catalogaron con sobrepeso y solo dos $(15.38 \%)$ con 


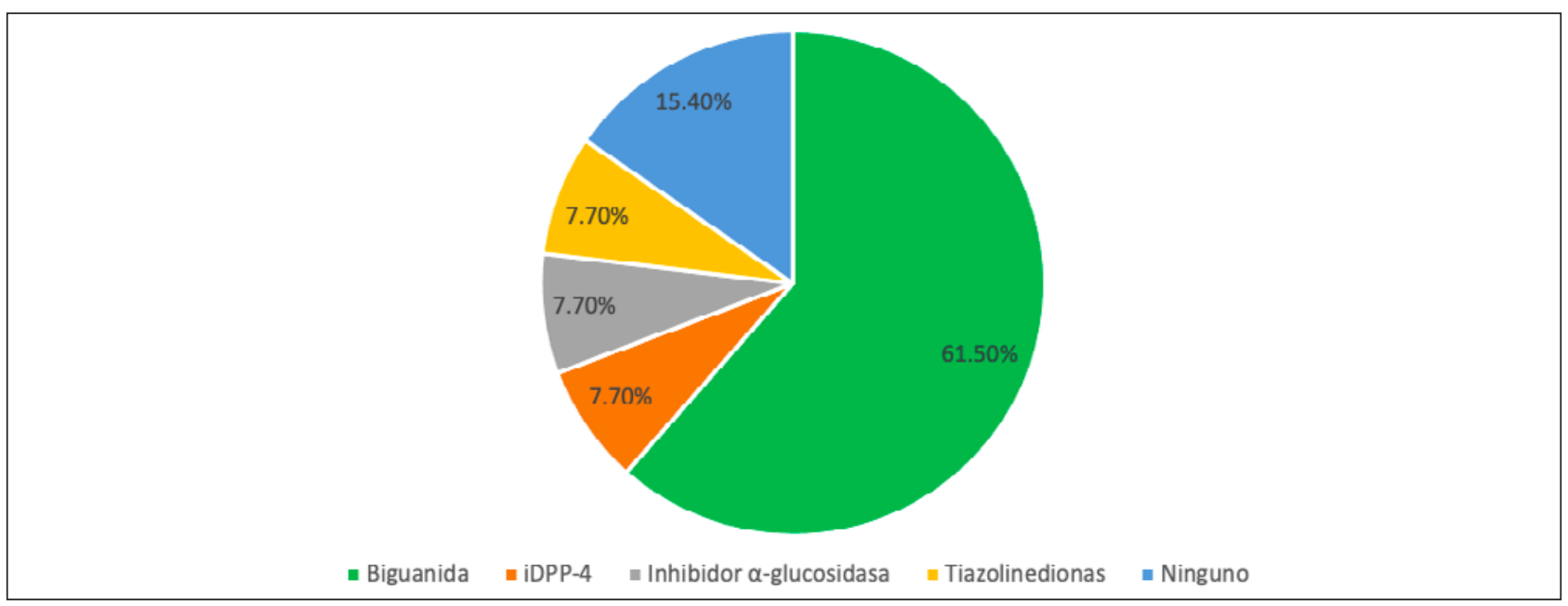

Figura 1. Tratamiento antidiabético oral previo a antivirales de acción directa. iDDP-4: inhibidores de la dipeptidil peptidasa 4.

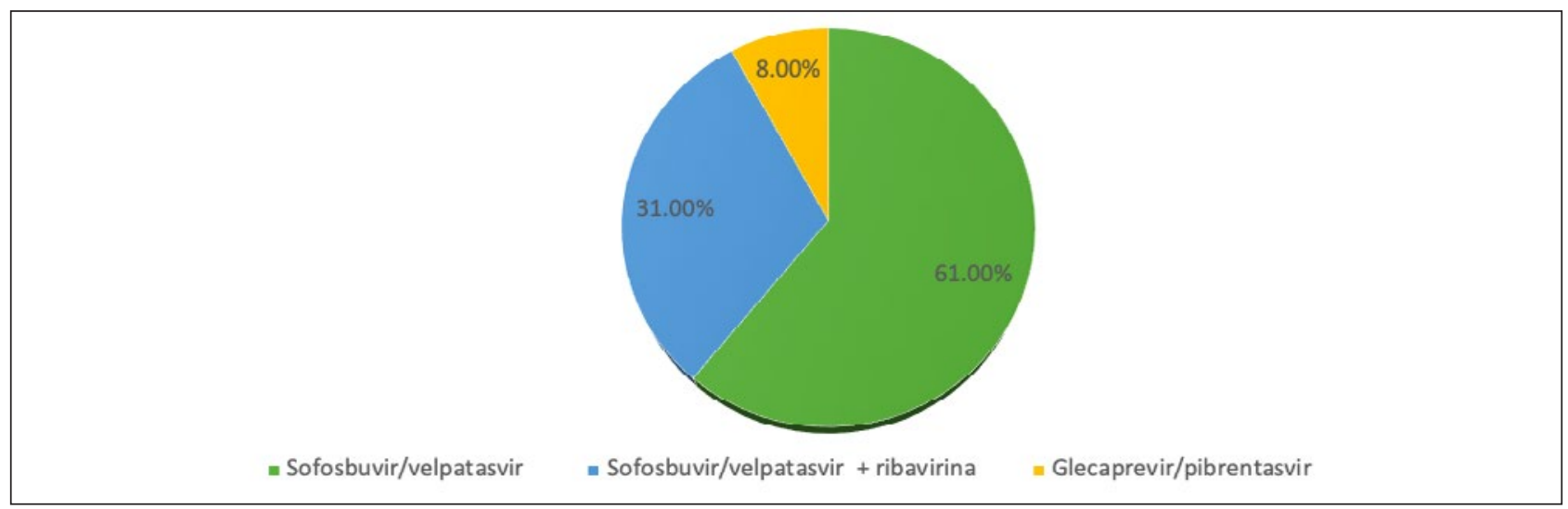

Figura 2. Tratamiento con antivirales de acción directa otorgado.

obesidad grado II. Se pueden observar las características basales de nuestra población en la tabla 1 .

Dentro del tratamiento para DM2, se concluyó que once $(84.6 \%)$ de los pacientes refirió apego a su tratamiento (mediante interrogatorio directo); también destaca que once pacientes (84.6\%) utilizaban antidiabéticos orales previo al inicio de los $A A D$, de los cuales el más utilizado fue la metformina en ocho (61.5\%) de los pacientes, podemos observar dicha distribución en la figura 1. El tratamiento antidiabético se inició en otro paciente posterior al término de la terapia con $A A D$, con lo cual doce (92.3\%) de los pacientes requirieron manejo para dicha comorbilidad. Respecto al uso de insulina previo al inicio de $A A D$ y posterior a esto, solo tres y cuatro pacientes (23.1 y $30.8 \%$ respectivamente) recibían dicho manejo.

\section{Características de la enfermedad hepática}

Todos los participantes del estudio cumplieron con la definición de infección crónica por VHC. Dentro de sus características destaca el tiempo de diagnóstico, siendo heterogéneo el tiempo de evolución, con un mínimo de seis y máximo de 324 meses (media: $94.54 \pm 101.113$ ), así como la carga viral al diagnóstico con media de 8,316,306 UI/ml. Dentro del genotipo se encontró que el más frecuente fue el numero 2, con ocho pacientes (61.5\%), y en solo uno de los pacientes se contaba con genotipo pero no el subtipo, ya que a partir de la llegada del tratamiento pangenotipo ya no es necesario obtener el genotipo, solo la carga viral. Se realizó la evaluación funcional de la insuficiencia hepática de acuerdo con la escala de Child-Pugh en su primera 
Tabla 2. Efecto metabólico del tratamiento antiviral directo

\begin{tabular}{lcc}
\hline Parámetro & Valores & Significancia $(\mathrm{p})$ \\
\hline Glucosa previo AAD; $(\mathrm{mg} / \mathrm{dl}) ; \mathrm{M} \pm \mathrm{DE}$ & $166.15 \pm 66.67$ & 0.008 \\
\hline Glucosa posterior AAD; $(\mathrm{mg} / \mathrm{dl}) ; \mathrm{M} \pm \mathrm{DE}$ & $123.31 \pm 26.97$ & 0.200 \\
\hline HbA1c previo AAD $(\%) ; \mathrm{M} \pm \mathrm{DE}$ & $7.62 \pm 2.32$ & 0.110 \\
\hline HbA1c posterior AAD $(\%) ; \mathrm{M} \pm \mathrm{DE}$ & $7.09 \pm 2.00$ & 0.200 \\
\hline & Correlación & Significancia bilateral \\
\hline HbA1C previo AAD y HbA1c posterior AAD* & 0.706 & 0.062 \\
\hline Glucosa previo AAD y glucosa posterior AAD* & 0.097 & 0.046 \\
\hline *Prueba t de Student. & & \\
AAD: antivirales de acción directa; HbA1c: hemoglobina glucosilada; DE: desviación estándar; M: media. &
\end{tabular}

consulta. Destaca que la mayoría de los pacientes presentaba una enfermedad hepática compensada, ya que nueve de ellos (69.2\%) fueron catalogados como Child-Pugh A, seguido de Child-Pugh B y C en el resto de los participantes (15.4 y $15.4 \%$ respectivamente).

\section{Tratamiento antiviral directo recibido y la respuesta viral sostenida}

Respecto al tratamiento antiviral directo, podemos observar en la figura 2 qué tratamiento se otorgó a los pacientes: ocho pacientes (61.5\%) recibieron sofosbuvir/velpatasvir, seguido de cuatro (30.8\%) que recibieron sofosbuvir/velpatasvir más ribavirina y a solo un paciente $(7.7 \%)$ se le otorgó un régimen con glecaprevir/pibrentasvir. En cuanto a la RVS, el 100\% (trece pacientes) alcanzó RVS, ya que contaban con carga viral de VHC indetectable doce semanas posteriores a concluir tratamiento antiviral.

\section{Efecto metabólico del tratamiento antiviral directo}

El objetivo principal de nuestro estudio fue evaluar el comportamiento del control glucémico. Se observó en nuestra población de estudio que los participantes presentaban media de glucosa sérica en ayuno de $166.15 \mathrm{mg} / \mathrm{dl}$; esta glucosa sérica correlaciona con los niveles de $\mathrm{HbA} 1 \mathrm{c}$, la cual se encontró una media del 7.62\%, previo al inicio del tratamiento con AAD. Se evaluaron los niveles de glucosa sérica y $\mathrm{HbA} 1 \mathrm{c}$ al término del tratamiento con $\mathrm{AAD}$, observando que la población de estudio presentó disminución significativa $(p=0.046)$ en los niveles de glucosa sérica en ayuno $(123.31 \mathrm{mg} / \mathrm{dl})$ y en la HbA1c (6.86\%), pero en este valor no alcanzó la significancia estadística ( $p=0.062$ ), esto se puede observar en los datos descritos en la tabla 2 .

El análisis del efecto del tratamiento antes y después se realizó con prueba t de Student, obteniendo diferencia significativa en la glucosa sérica en ayuno $(p=0.046)$; en la HbA1c hubo disminución, pero no alcanzó significancia estadística. Se muestra este resultado en la tabla 2.

\section{DISCUSIÓN}

En el presente estudio se demostró el impacto de los AAD en pacientes con diabetes mellitus e infección crónica por VHC en el Hospital de Especialidades Centro Médico Nacional Siglo XXI, ubicado en la Ciudad de México. En los pacientes reclutados en este estudio predominó el sexo femenino, lo cual se correlaciona con la ENSANUT 2016, donde la prevalencia de diabetes mellitus fue mayor en mujeres que en hombres (10.3 vs. $8.4 \%$ ).

Es importante destacar que la mayoría de los pacientes (84.6\%) presentó apego a su tratamiento antidiabético. Sin embargo, esto no se relacionó a sus niveles de glucosa sérica y $\mathrm{HbA} 1 \mathrm{c}$; esto podría ser explicado por la infección crónica por VHC, ya 
que dicha enfermedad es la que presenta una mayor tasa de resistencia a la insulina en comparación con otras enfermedades hepáticas crónicas o que no hubiera apego a la dieta indicada.

De acuerdo con lo reportado en estudios previos, los genotipos de VHC varían de un país a otro, sin embargo el genotipo 1 es el más común en nuestro país, y de los subtipos el $1 \mathrm{~b}$ es el de mayor prevalencia; mientras que en nuestra población el genotipo más frecuente fue el número 2 (61.5\%); la frecuencia del genotipo 2 en nuestro estudio se compara a un análisis de casos y controles en EE.UU. donde este se encontraba presente en el $29 \%$ de los pacientes diabéticos con infección crónica por VHC, y en Japón los pacientes con genotipo 2 tienen una tasa más alta de DM que el resto de genotipos; esto no se ha demostrado de forma consistente en otros estudios. Una de las razones por las que el genotipo 2 predominó en nuestro estudio se explica por el tiempo en que se realizó el reclutamiento, ya que en enero de 2019 se introdujo al esquema de tratamiento los AAD pangenotipo y previamente no contábamos con opciones de tratamiento para este genotipo; así también podría tratarse de un sesgo en la selección en población de personas con diabetes.

El objetivo principal de la terapia antiviral es la erradicación del ARN del VHC, lo cual se logra mediante la RVS, y dicha respuesta se alcanzo en el $100 \%$ de los participantes independientemente del tratamiento antiviral otorgado. El objetivo de nuestro estudio fue demostrar el impacto de la terapia con AAD en el control glucémico de los pacientes, ya que algunos ensayos clínicos han mostrado mejora en el control glucémico en personas con diabetes y sin diabetes posterior al tratamiento antiviral directo. En 2017 Hum, et al. realizaron un estudio en personas con diabetes e infección crónica por VHC donde se concluyó que la erradicación del VHC conduce a un mejor control glucémico en dicha población. Lo anterior correlaciona con los resultados de nuestro estudio, ya que todos los participantes tenían RVS y se demostró mejoría de los niveles de glucosa sérica (166.15 vs. 123.81 $\mathrm{mg} / \mathrm{dl}$ ), así como de $\mathrm{HbA} 1 \mathrm{c}$ (7.62 vs. $6.86 \%$ ), y al realizar el análisis por pares con prueba t de Student el resultado fue estadísticamente significativo para los niveles de glucosa sérica $(p=0.046)$, pero no fue así con el análisis de la HbA1c. Consideramos que un área de oportunidad para el estudio es incluir mayor número de participantes y determinar el impacto de la dieta en los resultados. La limitación principal de nuestro estudio es el número de participantes incluidos en la muestra, así como el tiempo de seguimiento de los pacientes, ya que el seguimiento a largo plazo impactaría en observar si la mejoría observada en el control glucémico se mantiene o solo es un efecto transitorio de la terapia antiviral. Sin embargo, la fortaleza principal es la falta de estudios en población mexicana y la asociación de dichas enfermedades.

\section{CONCLUSIÓN}

Con el tratamiento con AAD se observó mejoría de los niveles de glucosa sérica, así como de $\mathrm{HbA} 1 \mathrm{c}$ en los pacientes con infección crónica por VHC. Existe diferencia estadísticamente significativa para los niveles de glucosa sérica $(p=0.046)$, no así con la HbA1c $(p=0.062)$.

Hasta el momento no se habían realizado estudios en nuestro país sobre el impacto que tienen los AAD sobre el control glucémico en personas con diabetes, por lo cual este trabajo representa un acercamiento para demostrar dicho impacto. Respecto a estudios internacionales, es concordante la eficacia en la erradicación del ARN de VHC con el tratamiento antiviral directo, debido a que el $100 \%$ de los participantes presentó RVS independientemente del tiempo de evolución de la infección por VHC, carga viral, genotipo y clasificación funcional, debido a que todos los participantes presentaron RVS, lo cual condujo también a observar mejoría en los niveles de glucosa sérica en ayuno y niveles de $\mathrm{HbA} 1 \mathrm{c}$, que a largo plazo se han asociado a la aparición de complicaciones microvasculares y macrovasculares.

\section{FINANCIAMIENTO}

La presente investigación no ha recibido ninguna beca específica de agencias de los sectores públicos, comercial o sin ánimo de lucro. 


\section{CONFLICTO DE INTERESES}

Los autores declaran no tener conflicto de intereses.

\section{RESPONSABILIDADES ÉTICAS}

Protección de personas y animales. Los autores declaran que para esta investigación no se han realizado experimentos en seres humanos ni en animales.

Confidencialidad de los datos. Los autores declaran que han seguido los protocolos de su centro de trabajo sobre la publicación de datos de pacientes.

Derecho a la privacidad y consentimiento informado. Los autores han obtenido el consentimiento informado de los pacientes y/o sujetos referidos en el artículo. Este documento obra en poder del autor de correspondencia.

\section{BIBLIOGRAFÍA}

1. Desbois AC, Cacoub P. Diabetes mellitus, insulin resistance and hepatitis C virus infection: A contemporary review. World J Gastroenterol. 2017;23(9):1697-711.

2. Chiquete E, Ochoa-Guzmán A, García-Lamas L, Anaya-Gómez F, Gutiérrez-Manjarrez Jl, Sánchez-Orozco LV, et al. Hepatitis C virus infection and type 2 diabetes mellitus in Mexican patients. Rev Med Inst Mex Seguro Soc. 2012;50(5):481-6.

3. Ulises M, Marcelino M. Genotipos del virus de la hepatitis C, distribucion en adultos. Rev Med Inst Mex Seguro Soc. 2012;50(5):493-5.

4. Vanni E, Bugianesi E, Saracco G. Treatment of type 2 diabetes mellitus by viral eradication in chronic hepatitis C: myth or reality? Dig Liver Dis. 2016;48(2):105-11.

5. Negro F. Facts and fictions of HCV and comorbidities: steatosis, diabetes mellitus, and cardiovascular diseases. J Hepatol. 2014;61(1):S69-S78.

6. Burman B, Bacchetti P. Liver inflammation is a risk factor for prediabetes in at-risk latinos with and without hepatitis $C$ infection. Liver Int. 2015;35(1):101-7.

7. Hammerstad S,Grock S. Diabetes and hepatitis C: A two-way association. Front Endocrinol (Lausanne). 2015;6:134.

8. Stine JG, Wynter JA. Effect of treatment with direct acting antiviral on glycemic control in patients with diabetes mellitus and chronic hepatitis C. Ann Hepatol. 2017;16(2):215-20.

9. Okuroglu N, Sertbas M, Ozdemir A. Treatment of chronic hepatitis C can improve glycemic control in patients with type 2 diabetes. Case Reports Hepatol. 2018;2018:5260510.

10. Hum J, Jou JH. Improvement in glycemic control of type 2 diabetes after successful treatment of hepatitis C virus. Diabetes Care. 2017;40(9): 1173-80.

11. Sylvia D, Jakub $G$. Chronic hepatitis $C$ association with diabetes mellitus and cardiovascular risk in the era of DAA therapy. Can J Gastroenterol Hepatol. 2018;2018:6150861.

12. Holmes JA, Rutledge SM, Chung RT. Direct-acting antiviral treatment for hepatitis C. Lancet. 2019;393(10179):1392-4. 